\title{
On achieving size-independent stability margin of vehicular lattice formations with distributed control
}

\author{
He Hao, Prabir Barooah
}

\begin{abstract}
We study the stability margin of a vehicular formation with distributed control, in which the control at each vehicle only depends on the information from its neighbors in an information graph. We consider a D-dimensional lattice as information graph, of which the 1-D platoon is a special case. The stability margin is measured by the real part of the least stable eigenvalue of the closed-loop state matrix, which quantifies the rate of decay of initial errors. In [1], it was shown that with symmetric control, in which two neighbors put equal weight on information received from each other, the stability margin of a 1-D vehicular platoon decays to 0 as $O\left(1 / N^{2}\right)$, where $N$ is the number of vehicles. Moreover, a perturbation analysis was used to show that with vanishingly small amount of asymmetry in the control gains, the stability margin scaling can be improved to $O(1 / N)$. In this paper, we show that, with judicious choice of non-vanishing asymmetry in control, the stability margin of the closed loop can be bounded away from zero uniformly in $N$. Asymmetry in control gains thus makes the control architecture highly scalable. The results are also generalized to $D$-dimensional lattice information graphs that were studied in [2], and the correspondingly stronger conclusions than those derived in [2] are obtained. In addition, we show that the size-independent stability margin can be achieved with relative position and relative velocity (RPRV) feedback as well as relative position and absolute velocity (RPAV) feedback, while the analysis in [1], [2] was only for the RPAV case.
\end{abstract}

\section{Index Terms}

Asymmetric control, automated platoon, distributed control, multi-agent system, stability margin.

He Hao and Prabir Barooah are with Department of Mechanical and Aerospace Engineering, University of Florida, Gainesville, FL 32611, USA. This work was supported by the National Science Foundation through Grant CNS-0931885 and ECCS-0925534. 


\section{INTRODUCTION}

We study cooperative control of a large vehicular formation with distributed control. The vehicles are modeled as double integrators, and the control action at each vehicle is computed based on information from its neighbors, where the neighbor relationship is characterized by a lattice information graph. The control objective is to make the vehicular formation track a constant-velocity type desired trajectory while maintaining pre-specified constant separation among neighbors. The desired trajectory of the entire vehicular formation is given in terms of trajectories of a set of fictitious reference vehicles.

The problem of distributed control for multi-agent coordination is relevant to many applications such as automated highway system, collective behavior of bird flocks and animal swarms, and formation flying of unmanned aerial and ground vehicles for surveillance, reconnaissance and rescue, etc. [3]-[8]. A typical issue faced in distributed control is that as the number of agents increases, the performance (stability margin and sensitivity to external disturbances) of the closed loop degrades. Several recent papers have studied the scaling of performance of vehicle formations as a function of the number of vehicles. The references [1], [2] have studied the scaling of the stability margin of $D$-dimensional lattice formations. The stability margin is defined as the absolute value of the real part of the least stable eigenvalue of the closed loop. The stability margin characterizes the rate at which initial errors decay. The references [9]-[13] have examined the sensitivity of 1-dimensional platoons to external disturbances. However, among papers that examined sensitivity to disturbance, to the best of our knowledge only [13] has considered asymmetric control, the rest are limited to symmetric control. The control is called symmetric if between two neighboring vehicles $i$ and $j$, the weight $i$ puts on the information from $j$ is the same as the weight $j$ puts on the information from $i$.

In previous works on 1-D vehicular platoons, two types of feedback are respectively considered: relative position absolute velocity (RPAV) feedback [1], [12] and relative position relative velocity (RPRV) feedback [11], [13], [14]. With symmetric control, the stability margin of the vehicular platoon decays to 0 as $O\left(1 / N^{2}\right)$ in both types of feedback. This result for RPAV feedback was shown in [1], and for RPRV feedback was shown in [14]. The loss of stability margin with symmetric control has also been recognized by other researchers [12], [15]. Asymmetric control in the RPAV case was examined in [1], [2], where it was also shown 
that with vanishingly small asymmetry in the control gains, the stability margin can be improved to $O(1 / N)$. Similar conclusions are also obtained for a vehicle formation with a $D$-dimensional lattice as its information graph [2] - that decay of stability margin can be improved with asymmetry. In case of RPRV feedback, a similar improvement to $O(1 / N)$ with asymmetry was shown in [14], where only the relative velocity feedback gains were made asymmetric. The analyses in [1], [2], [14] were based on a partial differential equation (PDE) approximation of the closed loop dynamics and a perturbation method; the latter limited the results to only vanishingly small asymmetry.

In this paper we provide a stronger result on the stability margin with asymmetric control by avoiding the perturbation analysis of the aforementioned papers. We also avoid the PDE approximation and analyze the state space model directly. In particular, we show that with judicious choice of asymmetry in the control, the stability margin of the vehicular formation can be uniformly bounded away from 0 (independent of $N$ ) and derive a closed-form formula for the lower bound. This result makes it possible to design the control gains so that the stability margin of the system satisfies a pre-specified value irrespective of how many vehicles are in the formation. We also generalize the result to formations with $D$-dimensional information graphs, and show that a similar, size-independent stability margin can be obtained by using asymmetry in the control gains. These results are established for both RPAV and RPRV feedbacks.

The focus of this paper is on the stability margin, which is related to exponential stability of the closed loop system. A related concept is that of "string stability" [16]. String stability is usually interpreted as the system's sensitivity to external disturbances; see [6], [10], [17], [18] and references therein. We do not study sensitivity to external disturbances in this paper.

For ease of description, we first present the problem statement and main result for a vehicular formation with 1-dimensional information graph (i.e. the vehicular platoon) in Section II Analysis of the stability margin and numerical verification appear in Section III. The extension of the result to a vehicular formation with $D$-dimensional lattice information graph is presented in Section IV. The paper ends with a summary in Section V. 


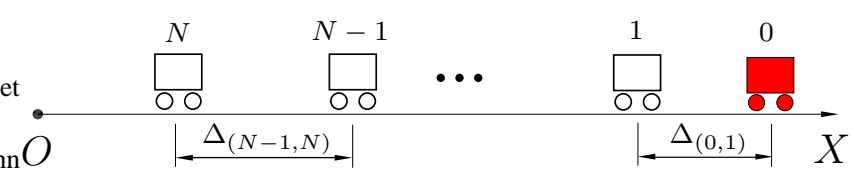

Fig. 1. Desired geometry of a vehicular platoon with $N$ vehicles and 1 "fictitious" reference vehicle. The filled vehicle in the front of the platoon represents the reference vehicle, it is denoted by index 0 .

\section{PRoblem STATEMENT AND RESUlt FOR 1-D PLATOON}

\section{A. Problem statement}

In this section we consider the formation control of $N$ homogeneous vehicles which are moving in 1-D Euclidean space, as shown in Figure 1. The position of the $i$-th vehicle is denoted by $p_{i} \in \mathbb{R}$ and the dynamics of each vehicle are modeled as a double integrator:

$$
\ddot{p}_{i}=u_{i}, \quad i \in\{1,2, \cdots, N\}
$$

where $u_{i} \in \mathbb{R}$ is the control input. This is a commonly used model for vehicle dynamics in studying vehicular formations, which results from feedback linearization of non-linear vehicle dynamics [19], [20].

The control objective is that vehicles maintain a desired formation geometry while following a constant-velocity type desired trajectory. The desired geometry of the formation is specified by the desired gaps $\Delta_{(i-1, i)}$ for $i \in\{1, \cdots, N\}$, where $\Delta_{(i-1, i)}$ is the desired value of $p_{i-1}(t)-p_{i}(t)$. The desired inter-vehicular gaps $\Delta_{(i-1, i)}$ 's are positive constants and they have to be specified in a mutually consistent fashion, i.e. $\Delta_{(i, k)}=\Delta_{(i, j)}+\Delta_{(j, k)}$ for every triple $(i, j, k)$ where $i \leq j \leq k$. The desired trajectory of the platoon is provided in terms of a fictitious reference vehicle with index 0 , whose trajectory is given by $p_{0}^{*}(t)=v^{*} t+c_{0}$ for some constants $v^{*}, c_{0}$, where $v^{*}$ is the cruise velocity of the formation. The desired trajectory of the $i$-th vehicle, $p_{i}^{*}(t)$, is given by $p_{i}^{*}(t)=p_{0}^{*}(t)-\Delta_{(0, i)}=p_{0}^{*}(t)-\sum_{j=1}^{i} \Delta_{(j-1, j)}$.

We consider the following distributed control laws.

1) Relative position and absolute velocity (RPAV) feedback: the control action at the $i$-th vehicle depends on the relative position measurements with its two neighbors (one on either 
side), its own velocity, and the desired velocity $v^{*}$ :

$$
\begin{aligned}
u_{i}= & -k_{i}^{f}\left(p_{i}-p_{i-1}+\Delta_{(i-1, i)}\right)-k_{i}^{b}\left(p_{i}-p_{i+1}-\Delta_{(i, i+1)}\right) \\
& -b_{i}\left(\dot{p}_{i}-v^{*}\right), \quad i \in\{1, \cdots, N-1\}, \\
u_{N}= & -k_{N}^{f}\left(p_{N}-p_{N-1}+\Delta_{(N-1, N)}\right)-b_{N}\left(\dot{p}_{N}-v^{*}\right),
\end{aligned}
$$

where $k_{i}^{f}, k_{i}^{b}$ are the front and back position gains and $b_{i}$ is the velocity gain.

2) Relative position and relative velocity $(R P R V)$ feedback: the control action at the $i$-th vehicle depends on the relative position and relative velocity measurements with its nearest neighbors in the platoon:

$$
\begin{aligned}
u_{i}= & -k_{i}^{f}\left(p_{i}-p_{i-1}+\Delta_{(i-1, i)}\right)-k_{i}^{b}\left(p_{i}-p_{i+1}-\Delta_{(i, i+1)}\right) \\
& -b_{i}^{f}\left(\dot{p}_{i}-\dot{p}_{i-1}\right)-b_{i}^{b}\left(\dot{p}_{i}-\dot{p}_{i+1}\right), \quad i \in\{1, \cdots, N-1\}, \\
u_{N}= & -k_{N}^{f}\left(p_{N}-p_{N-1}+\Delta_{(N-1, N)}\right)-b_{N}^{f}\left(\dot{p}_{N}-\dot{p}_{N-1}\right),
\end{aligned}
$$

where $k_{i}^{f}, k_{i}^{b}$ (respectively, $b_{i}^{f}, b_{i}^{b}$ ) are the front and back position (respectively, velocity) gains of the $i$-th vehicle.

In the RPRV feedback case, vehicle $i$ must be provided (a-priori) the desired gaps with its two neighbors. In the RPAV feedback, it must be provided with additional information: the formation's desired velocity $v^{*}$. The closed-loop dynamics with RPAV (resp., RPRV) feedback, in terms of the tracking errors $\tilde{p}_{i}:=p_{i}-p_{i}^{*}$, can now be expressed as:

$$
\dot{x}=A^{(\mathrm{RPAV})} x, \quad \text { (resp.) } \quad \dot{x}=A^{(\mathrm{RPRV})} x,
$$

where the state vector is defined as $x:=\left[\tilde{p}_{1}, \dot{\tilde{p}}_{1}, \cdots, \tilde{p}_{N}, \dot{\tilde{p}}_{N}\right] \in \mathbb{R}^{2 N}$, and the state matrix $A^{(.)}$ depends on the control gains but not on the desired gaps or desired velocity.

Definition 1: The stability margin $S^{(\mathrm{RPAv})}$ (respectively, $\left.S^{(\mathrm{RPRV})}\right)$ of the closed-loop system (4) is defined as the absolute value of the real part of the least stable eigenvalue of $A^{(\mathrm{RPAV})}$ (respectively, $A^{(\mathrm{RPRV})}$ ). The control law (2) (respectively, (3)) is symmetric if each vehicle uses the same front and back control gains: $k_{i}^{f}=k_{i}^{b}=k_{0}, b_{i}=b_{0}$ (respectively, $k_{i}^{f}=k_{i}^{b}=k_{0}, b_{i}^{f}=b_{i}^{b}=b_{0}$ ), for all $i \in\{1,2, \cdots, N-1\}$, where $k_{0}, b_{0}$ are positive constants.

In this paper, we consider the following asymmetric control gains

$$
\text { RPAV feedback: } k_{i}^{f}=(1+\epsilon) k_{0}, \quad k_{i}^{b}=(1-\epsilon) k_{0}, \quad b_{i}=b_{0} .
$$




$$
\begin{array}{ll}
\text { RPRV feedback: } & k_{i}^{f}=(1+\epsilon) k_{0}, \quad k_{i}^{b}=(1-\epsilon) k_{0}, \\
& b_{i}^{f}=(1+\epsilon) b_{0}, \quad b_{i}^{b}=(1-\epsilon) b_{0},
\end{array}
$$

where $\epsilon \in[0,1)$ denotes the amount of asymmetry; $\epsilon=0$ corresponds to symmetric control. The design for the RPAV case is inspired by [1], [2]. The control gains given in (5) and (6) are homogeneous in the sense that they do not vary with $i$. The reason we only consider homogeneous control gains is that heterogeneity has little effect on the scaling of stability margin, see [14] for a proof for 1-D platoon. The proof for vehicular formation with general graphs is given in Lemma 2, which is provided in the appendix.

The following proposition summaries the results in [1], [14].

Proposition 1: Consider an $N$-vehicle platoon with closed loop dynamics (4).

1) [Corollary 1 of [1], Theorem 1 of [14]] With symmetric control $(\epsilon=0)$, both $S^{(\mathrm{RPAV})}$ and $S^{(\mathrm{RPRV})}$ are $O\left(\frac{1}{N^{2}}\right)$.

2) [Corollary 3 of [1]] With the asymmetric control gains $k_{i}^{f}=k_{0}(1+\epsilon), k_{i}^{b}=k_{0}(1-\epsilon)$ and $b_{i}=b_{0}$, the stability margin of the platoon with RPAV feedback is $S^{(\mathrm{RPAV})}=O\left(\frac{\epsilon}{N}\right) .1$

3) [Theorem 2 of [14]] With asymmetric control gains $k_{i}^{f}=k_{i}^{b}=k_{0}, b_{i}^{f}=b_{0}(1+\epsilon), b_{i}^{b}=$ $b_{0}(1-\epsilon)$, the stability margin of the platoon with RPRV feedback is $S^{(\mathrm{RPRV})}=O\left(\frac{\epsilon}{N}\right)$.

Statements (2) and (3) hold in the limit $\epsilon \rightarrow 0$ and $N \rightarrow \infty$.

Proposition 1 shows that with symmetric control, the stability margin decays to 0 as $O\left(1 / N^{2}\right)$, irrespective of the type of feedback we used. However, in the case of RPAV feedback, with vanishingly small amount of asymmetry in the position gains, the stability margin of the system can be improved to $O(1 / N)$. The same $O(1 / N)$ trend can be achieved for the case of RPRV feedback with vanishingly small asymmetry in the velocity gains alone while the position gains are held symmetric. The design (6) was not considered in [14]. Since the results in [1], [14] were obtained with a perturbation analysis, these results are applicable only when the amount of asymmetry is vanishingly small.

The following theorem is the main result of this paper, whose proof and numerical corroboration are given in Section $\amalg$,

\footnotetext{
${ }^{1}$ The case considered in [1] was that $\left|k_{i}^{f}-k_{0}\right|<\epsilon,\left|k_{i}^{b}-k_{0}\right|<\epsilon$. It is straightforward, however, to re-derive the results if the constraints on the gains are changed to the form used here: $\left|k_{i}^{f}-k_{0}\right| / k_{0}<\epsilon,\left|k_{i}^{b}-k_{0}\right| / k_{0}<\epsilon$. In this paper we consider the latter case since it makes the analysis cleaner without changing the results of [1] significantly.
} 
Theorem 1: With the control gains given in (5) and (6) respectively, for any fixed $\epsilon \in(0,1)$, the closed loop is exponentially stable and the stability margin of the vehicular platoon is bounded away from 0 uniformly in $N$. Specifically,

$$
\begin{aligned}
& S^{(\mathrm{RPAV})} \geq \frac{\Re\left(b_{0}-\sqrt{b_{0}^{2}-8 k_{0}\left(1-\sqrt{1-\epsilon^{2}}\right)}\right)}{2}, \\
& S^{(\mathrm{RPRV})} \geq \min \left\{b_{0}\left(1-\sqrt{1-\epsilon^{2}}\right), \frac{k_{0}}{b_{0}}\right\},
\end{aligned}
$$

where $\Re($.$) denotes the real part.$

Remark 1: Comparing Theorem 1 with Proposition 1, we observe the following: (1) Even with an arbitrarily small (but fixed and non-vanishing) amount of asymmetry in the control gains, the stability margin of the system can be bounded away from zero uniformly in $N$. This asymmetric design therefore makes the resulting control law highly scalable; it eliminates the degradation of stability margin with increasing $N$. (2) In case of the RPAV feedback, although the control law is the same as that analyzed in [1], the stronger conclusion we obtained - compared to that in [1] - is due to the fact that our analysis does not rely on a perturbation-based technique that was used [1], which limited the analysis in [1] to vanishingly small $\epsilon$. (3) For the RPRV feedback case, the stronger result compared to that in [14], is obtained by putting equal asymmetry in both position and velocity gains, while [14] allowed asymmetry only in the velocity gain. In addition, unlike [1], [14], we do not use a PDE (partial differential equation) approximation to analyze the stability margin, but analyze the state-space model directly.

\section{StABILITy MARGIN OF THE 1-D VEHICULAR PLATOON}

With the control gains specified in (5) and (6) respectively, it can be shown that the state matrices can be expressed in the following forms,

$$
\begin{aligned}
& A^{(\mathrm{RPAV})}=I_{N} \otimes A_{1}+L^{(1)} \otimes A_{2}, \\
& A^{(\mathrm{RPRV})}=I_{N} \otimes A_{3}+L^{(1)} \otimes A_{4},
\end{aligned}
$$

where $I_{N}$ is the $N \times N$ identity matrix, $\otimes$ denotes the Kronecker product, and

$$
A_{1}:=\left[\begin{array}{cc}
0 & 1 \\
0 & -b_{0}
\end{array}\right], \quad A_{2}:=\left[\begin{array}{cc}
0 & 0 \\
-k_{0} & 0
\end{array}\right] \text {, }
$$




$$
A_{3}:=\left[\begin{array}{ll}
0 & 1 \\
0 & 0
\end{array}\right], \quad A_{4}:=\left[\begin{array}{cc}
0 & 0 \\
-k_{0} & -b_{0}
\end{array}\right],
$$

where $k_{0}>0, b_{0}>0$ are the nominal position and velocity gains respectively, and

$$
L^{(1)}:=\left[\begin{array}{cccc}
2 & -1+\epsilon & & \\
-1-\epsilon & 2 & -1+\epsilon & \\
& \ddots & \ddots & \ddots \\
& -1-\epsilon & 2 & -1+\epsilon \\
& & -1-\epsilon & 1+\epsilon
\end{array}\right] .
$$

It follows from Theorem 3.1 of [21] that the eigenvalues of $L^{(1)}$ are given by

$$
\lambda=b+2 c \rho \cos \theta
$$

if $\theta(\theta \neq m \pi, m \in \mathbb{Z}, \mathbb{Z}$ being the set of integers) is a solution to

$$
\begin{array}{r}
\rho^{N}(a c \sin (N+1) \theta+(\gamma \delta-\alpha \beta) \sin (N-1) \theta \\
-c \rho(\gamma+\delta) \sin N \theta)-\left(c \alpha \rho^{2 N}+a \beta\right) \sin \theta=0,
\end{array}
$$

where $a=-1-\epsilon, b=2, c=-1+\epsilon, \alpha=\beta=\gamma=0, \delta=-1+\epsilon, \rho=\sqrt{(-1-\epsilon) /(-1+\epsilon)}$. Eq. (12) and (13) can now be simplified to

$$
\lambda_{\ell}=2-2 \sqrt{1-\epsilon^{2}} \cos \theta_{\ell}, \quad \ell \in\{1,2, \cdots, N\},
$$

where $\epsilon \in(0,1)$ and $\theta_{\ell}$ is the $\ell$-th root of the following equation

$$
\sqrt{\frac{1+\epsilon}{1-\epsilon}} \sin (N+1) \theta=\sin N \theta .
$$

From (14), we see that the eigenvalues of $L^{(1)}$ are real and positive, and moreover, $0<\lambda_{1}=$ $2-2 \sqrt{1-\epsilon^{2}} \cos \theta_{1}<\lambda_{2}<\cdots<\lambda_{N}=2-2 \sqrt{1-\epsilon^{2}} \cos \theta_{N}$, where $\theta_{1} \in\left(\frac{\pi}{2(N+1)}, \frac{3 \pi}{2(N+1)}\right), \theta_{N} \in$ $\left(\frac{(2 N-1) \pi}{2(N+1)}, \frac{(2 N+1) \pi}{2(N+1)}\right)$ are the solutions to $(15)$. To see why, first notice that we only need consider the roots of (15) in the open interval $(0,2 \pi)$, in which there are $2 N$ nontrivial isolated roots. See Figure 2 for an example. The roots located in $\mathbb{R} \backslash(0,2 \pi)$ are $2 m \pi(m \in \mathbb{Z})$ distance away from those in $(0,2 \pi)$. Moreover, if $\theta_{0} \in(0,2 \pi)$ is a solution of $(15)$, then $2 \pi-\theta_{0}$ is also a solution. Therefore, we can restrict the domain of analysis to $(0, \pi)$, in which there are $N$ isolated roots. The ordering of the eigenvalues follows from $\cos \theta$ being a decreasing function in $(0, \pi)$. It is 


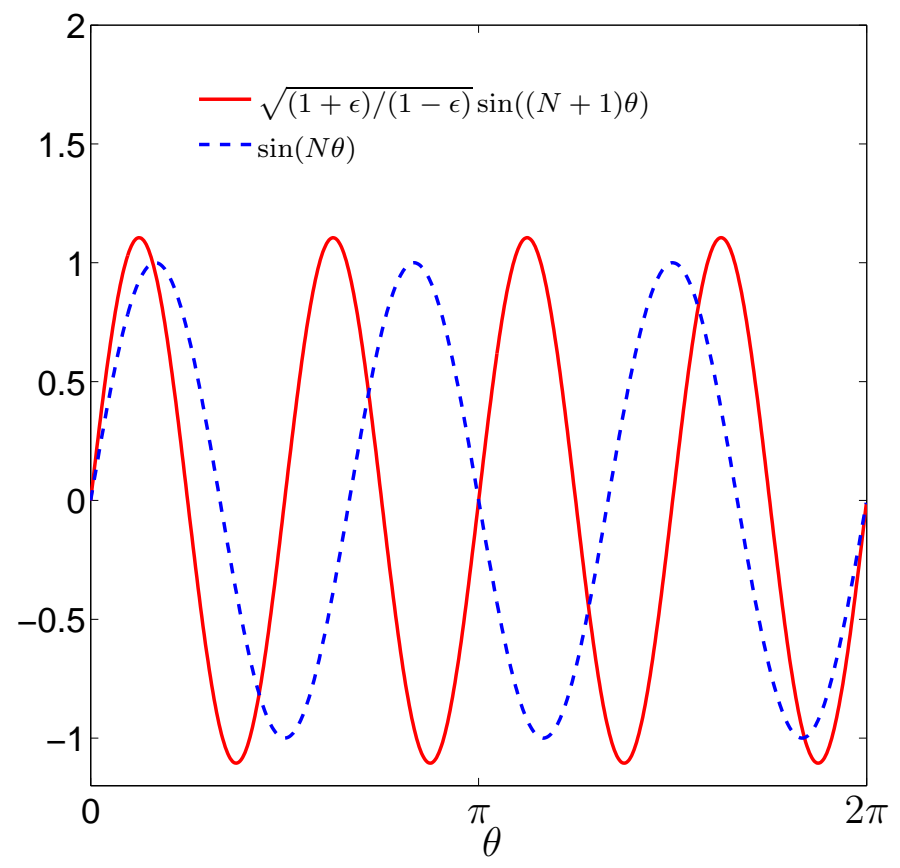

Fig. 2. Graphical solution $\theta$ of $\sqrt{(1+\epsilon) /(1-\epsilon)} \sin ((N+1) \theta)=\sin (N \theta)$ with $\epsilon=0.1$ and $N=3$.

straightforward to show from graphical solution of (15) that the $\ell$-th root $\theta_{\ell}$ is in the open interval $\left(\frac{(2 \ell-1) \pi}{2(N+1)}, \frac{(2 \ell+1) \pi}{2(N+1)}\right)$. We now present a formula for the stability margin of the vehicular platoon in terms of the eigenvalues of $L^{(1)}$.

Lemma 1: With the control gains given in (5) and (6) respectively, and $0<\epsilon<1$, the stability margin of the vehicular platoon is

$$
\begin{aligned}
& S^{(\mathrm{RPAV})}= \begin{cases}\frac{b_{0}}{2}, & \text { if } \lambda_{1} \geq b_{0}^{2} / 4 k_{0}, \\
\frac{b_{0}-\sqrt{b_{0}^{2}-4 k_{0} \lambda_{1}}}{2}, & \text { otherwise, }\end{cases} \\
& S^{(\mathrm{RPRV})}= \begin{cases}\frac{b_{0} \lambda_{1}}{2}, & \text { if } \lambda_{N} \leq 4 k_{0} / b_{0}^{2}, \\
\frac{2 k_{0}}{b_{0}+\sqrt{b_{0}^{2}-4 k_{0} / \lambda_{N}},} & \text { if } \lambda_{1} \geq 4 k_{0} / b_{0}^{2}, \\
\min \left\{\frac{b_{0} \lambda_{1}}{2}, \frac{2 k_{0}}{b_{0}+\sqrt{b_{0}^{2}-4 k_{0} / \lambda_{N}}}\right\}, & \text { otherwise, }\end{cases}
\end{aligned}
$$

where $\lambda_{1}$ and $\lambda_{N}$ are the smallest and largest eigenvalues of $L^{(1)}$ respectively.

Proof of Lemma 1] Our proof follows a similar line of attack as of [22]. From Schur's triangu- 
larization theorem, there exists an unitary matrix $U$ such that

$$
U^{-1} L^{(1)} U=L_{u}
$$

where $L_{u}$ is an upper-triangular matrix whose diagonal entries are the eigenvalues $\lambda_{\ell}$ of $L^{(1)}$. We first consider the RPAV feedback case. We do a similarity transformation on matrix $A^{(\mathrm{RPAV})}$.

$$
\begin{aligned}
\bar{A}^{(\mathrm{RPAV})} & :=\left(U^{-1} \otimes I_{2}\right) A^{(\mathrm{RPAV})}\left(U \otimes I_{2}\right) \\
& =\left(U^{-1} \otimes I_{2}\right)\left(I_{N} \otimes A_{1}+L^{(1)} \otimes A_{2}\right)\left(U \otimes I_{2}\right) \\
& =I_{N} \otimes A_{1}+L_{u} \otimes A_{2} .
\end{aligned}
$$

It is a block upper-triangular matrix, and the block on each diagonal is $A_{1}+\lambda_{\ell} A_{2}$, where $\lambda_{\ell} \in$ $\sigma\left(L^{(1)}\right)$, and $\sigma(\cdot)$ denotes the spectrum (the set of eigenvalues). Since similarity transformation preserves eigenvalues, and the eigenvalues of a block upper-triangular matrix are the union of eigenvalues of each block on the diagonal, we have

$$
\begin{aligned}
\sigma\left(A^{(\mathrm{RPAV})}\right)=\sigma\left(\bar{A}^{(\mathrm{RPAV})}\right) & =\bigcup_{\lambda_{\ell} \in \sigma\left(L^{(1)}\right)}\left\{\sigma\left(A_{1}+\lambda_{\ell} A_{2}\right)\right\} \\
& =\bigcup_{\lambda_{\ell} \in \sigma\left(L^{(1)}\right)}\left\{\sigma\left[\begin{array}{cc}
0 & 1 \\
-k_{0} \lambda_{\ell} & -b_{0}
\end{array}\right]\right\} .
\end{aligned}
$$

It follows now that the eigenvalues of $A^{(\mathrm{RAN})}$ are the roots of the characteristic equation $s^{2}+$ $b_{0} s+k_{0} \lambda_{\ell}=0$. For each $\ell \in\{1,2, \cdots, N\}$, the two roots are

$$
s_{\ell}^{ \pm}=\frac{-b_{0} \pm \sqrt{b_{0}^{2}-4 k_{0} \lambda_{\ell}}}{2} .
$$

The root closer to the imaginary axis is denoted by $s_{\ell}^{+}$, and is called the less stable eigenvalue between the two. The least stable eigenvalue is the one closet to the imaginary axis among them, it is denoted by $s_{\min }$. It follows from Definition 1 that $S=\left|\Re\left(s_{\min }\right)\right|$.

Depending on the discriminant in (17), there are two cases to analyze:

1) If $\lambda_{1} \geq b_{0}^{2} / 4 k_{0}$, due to $\lambda_{1}<\cdots<\lambda_{N}$, we have the discriminant in (17) for each $\ell$ is non-positive, which yields $S^{(\mathrm{RPAV})}=\left|\Re\left(s_{\min }\right)\right|=\frac{b_{0}}{2}$.

2) Otherwise, the less stable eigenvalues can be written as $s_{\ell}^{+}=\frac{1}{2}\left(-b_{0}+\sqrt{b_{0}^{2}-4 k_{0} \lambda_{\ell}}\right)$, which may be complex for some $\ell>1$. The least stable eigenvalue is obtained by setting $\lambda_{\ell}=\lambda_{1}$, so that $S^{(\mathrm{RPAV})}=\left|\Re\left(s_{\min }\right)\right|=\frac{1}{2}\left(b_{0}-\sqrt{b_{0}^{2}-4 k_{0} \lambda_{1}}\right)$. 
For the case of RPRV feedback, following the same procedure as that of RPAV feedback, the characteristic equations are given by

$$
s^{2}+\lambda_{\ell} b_{0} s+\lambda_{\ell} k_{0}=0 .
$$

For each $\ell \in\{1,2, \cdots, N\}$, the two roots of the characteristic equations (18) are,

$$
s_{\ell}^{ \pm}=-\frac{\lambda_{\ell} b_{0}}{2} \pm \frac{\sqrt{\left(\lambda_{\ell} b_{0}\right)^{2}-4 \lambda_{\ell} k_{0}}}{2} .
$$

Depending on the discriminant in (19), there are three cases to analyze:

1) If $\lambda_{N} \leq 4 k_{0} / b_{0}^{2}$, then the discriminant in (19) for each $\ell$ is non-positive. Recall that the stability margin is defined as the absolute value of the real part of the least stable eigenvalue, which yields

$$
S^{(\mathrm{RPRV})}=\left|\Re\left(s_{\min }\right)\right|=\frac{\lambda_{1} b_{0}}{2} .
$$

2) If $\lambda_{1} \geq 4 k_{0} / b_{0}^{2}$, then the discriminant in (19) for each $\ell$ is non-negative, the less stable eigenvalue can be written as

$$
s_{\ell}^{+}=-\frac{\lambda_{\ell} b_{0}-\sqrt{\left(\lambda_{\ell} b_{0}\right)^{2}-4 \lambda_{\ell} k_{0}}}{2}=-\frac{2 k_{0}}{b_{0}+\sqrt{b_{0}^{2}-4 k_{0} / \lambda_{\ell}}} .
$$

The least stable eigenvalue is achieved by setting $\lambda_{\ell}=\lambda_{N}$, then have the stability margin

$$
S^{(\mathrm{RPRV})}=\left|\Re\left(s_{\min }\right)\right|=\frac{2 k_{0}}{b_{0}+\sqrt{b_{0}^{2}-4 k_{0} / \lambda_{N}}} .
$$

3) Otherwise, if the discriminant in (19) is negative for small $\ell$ and positive for large $\ell$, then the stability margin is given by taking the minimum of the two cases above. This completes the proof.

We are now ready to present the proof of Theorem 1 .

Proof of Theorem 11 We see from Lemma 1 that the smallest and largest eigenvalues of matrix $L^{(1)}$ play important roles in determining the stability margin. To get a lower bound of the stability margin, a lower bound for the smallest eigenvalue and an upper bound for the largest eigenvalue is needed. Recall that $\lambda_{1}=2-2 \sqrt{1-\epsilon^{2}} \cos \theta_{1}, \lambda_{N}=2-2 \sqrt{1-\epsilon^{2}} \cos \theta_{N}$, where $\theta_{1} \in\left(\frac{\pi}{2(N+1)}, \frac{3 \pi}{2(N+1)}\right), \theta_{N} \in\left(\frac{(2 N-1) \pi}{2(N+1)}, \frac{(2 N+1) \pi}{2(N+1)}\right)$. We therefore have $\theta_{1} \rightarrow 0, \theta_{N} \rightarrow \pi$ as $N \rightarrow \infty$, and consequently,

$$
\inf _{N} \lambda_{1}=2-2 \sqrt{1-\epsilon^{2}}
$$




$$
\sup _{N} \lambda_{N}=2+2 \sqrt{1-\epsilon^{2}} \text {. }
$$

To prove the result with RPAV feedback, we consider the following two cases:

1) Case 1: $\lambda_{1} \geq b_{0}^{2} / 4 k_{0}$. According to Lemma 1, the stability margin is given by $S^{(\mathrm{RPAV})}=b_{0} / 2$.

2) Case 2: $\lambda_{1}<b_{0}^{2} / 4 k_{0}$. From Lemma 1, the stability margin is given by

$$
S^{(\mathrm{RPAV})}=\frac{b_{0}-\sqrt{b_{0}^{2}-4 k_{0} \lambda_{1}}}{2}
$$

Since $\lambda_{1} \geq 2-2 \sqrt{1-\epsilon^{2}}$, we obtain

$$
S^{(\mathrm{RPAV})} \geq \frac{b_{0}-\sqrt{b_{0}^{2}-8 k_{0}\left(1-\sqrt{1-\epsilon^{2}}\right)}}{2} .
$$

Notice that the above lower bound (22) is smaller than $b_{0} / 2$, the value of $S^{(\mathrm{RPAV})}$ in case 1 . The real part sign $\Re($.) in (7) comes from combining the above two cases. We obtain the first result of the theorem.

To prove the result with RPRV feedback, we consider the following three cases:

1) Case 1: $\lambda_{N} \leq 4 k_{0} / b_{0}^{2}$. According to Lemma 1, the stability margin is $S^{(\mathrm{RPRV})}=b_{0} \lambda_{1} / 2$. Moreover, from (20), we have $\inf _{N} \lambda_{1}=2-2 \sqrt{1-\epsilon^{2}}$, therefore the stability margin has the lower bound

$$
S^{(\mathrm{RPRV})} \geq b_{0}\left(1-\sqrt{1-\epsilon^{2}}\right) .
$$

2) Case 2: $\lambda_{1} \geq 4 k_{0} / b_{0}^{2}$. From Lemma 1, the stability margin is given by

$$
S^{(\mathrm{RPRV})}=\frac{2 k_{0}}{b_{0}+\sqrt{b_{0}^{2}-4 k_{0} / \lambda_{N}}} .
$$

In addition, we have from (21) that $\sup _{N} \lambda_{N}=2+2 \sqrt{1-\epsilon^{2}}$, so the stability margin for this case is bounded below as

$$
S^{(\mathrm{RPRV})} \geq \frac{2 k_{0}}{b_{0}+\sqrt{b_{0}^{2}-2 k_{0} /\left(1+\sqrt{1-\epsilon^{2}}\right)}} .
$$

3) Case 3: Otherwise, the stability margin are bounded below by the minimum of the above two cases.

Notice that in the second case, $\frac{2 k_{0}}{b_{0}+\sqrt{b_{0}^{2}-2 k_{0} /\left(1+\sqrt{1-\epsilon^{2}}\right)}} \geq \frac{k_{0}}{b_{0}}$. Combining the above three cases, we have that

$$
S^{(\mathrm{RPRV})} \geq \min \left\{b_{0}\left(1-\sqrt{1-\epsilon^{2}}\right), \frac{k_{0}}{b_{0}}\right\}
$$

which completes the proof. 


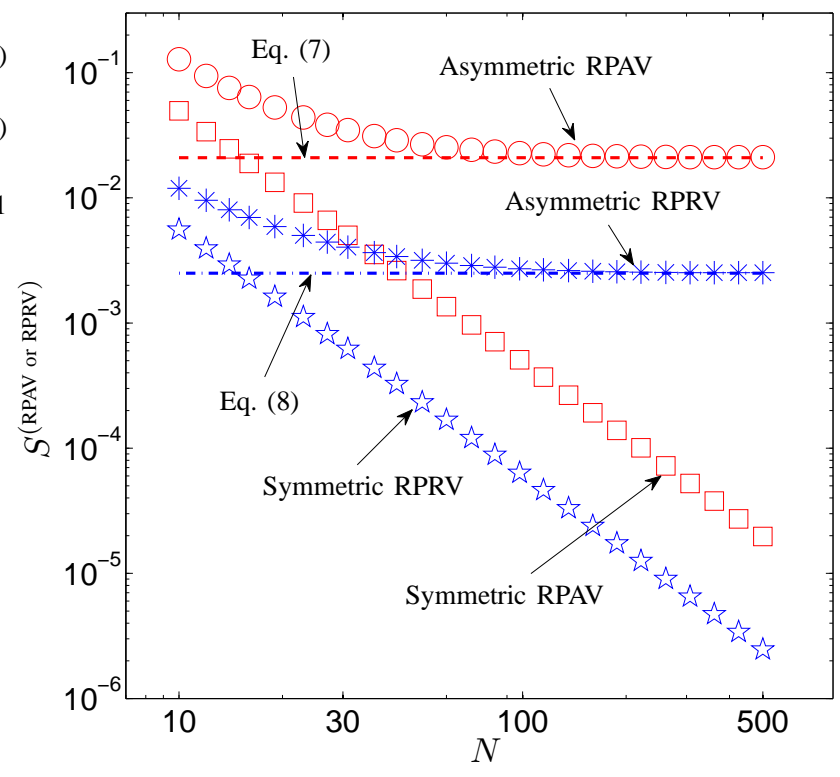

Fig. 3. Stability margin comparisons between symmetric control and asymmetric control.

\section{A. Numerical verification for 1-D vehicular platoon}

In this section, we present numerical verification of the lower bounds of the stability margins for both RPAV and RPRV feedbacks with asymmetric control, which are predicted by Theorem 1 . In addition, the stability margins with symmetric control are also computed to compare with the asymmetric case. The stability margins are obtained by numerically evaluating the eigenvalues of the state matrix $A^{(\mathrm{RPAV} \text { or RPRV) }}$ of (4) with corresponding controllers. Figure 3 depicts the comparisons between the stability margins with symmetric and asymmetric control for the two types of feedback: RPAV and RPRV. For both symmetric and asymmetric controls, the nominal control gains used are $k_{0}=1, b_{0}=0.5$, and for asymmetric control, the amount of asymmetry is $\epsilon=0.1$. We can see from Figure 3 that the stability margin of the vehicular platoon with asymmetric control is indeed bounded away from 0 uniformly in $N$, and the predictions Eq. (7) and Eq. (8) of Theorem 1 are quite accurate. Furthermore, for the same $N$, the stability margin with asymmetric control is much larger than that with symmetric control, especially when $N$ is large. 


\section{Stability MARGin With D-Dimensional LATTICE INFORMATion GRAPH}

In this section we analyze a more general scenario than the 1-D platoon of the previous sections. We consider a vehicular formation in which the position of each vehicle has dimension higher than one, such as a vehicular formation moving in 2-D or 3-D space. We assume the dynamics of each of the coordinates of a vehicle's position are decoupled and each coordinate can be independently controlled. Under this fully actuated assumption, the closed loop dynamics for each coordinate of the position can be independently studied; see [2], [6] for examples. The information used by a vehicle to compute its control is based on relative measurements with a set of neighbors specified in terms of an information graph. The problem formulation is similar to the 1-D case in the sense that each vehicle has to maintain constant separation with its neighbors in an information graph, except that the information graph now is a $D$-dimensional lattice.

Definition 2: An information graph is a graph $\mathbf{G}=(\mathbf{V}, \mathbf{E})$, where the set of nodes (vehicles) $\mathbf{V}=\left\{1,2, \ldots, N, N+1, \ldots, N+N_{r}\right\}$ consists of $N$ real vehicles and $N_{r}$ "fictitious" reference vehicles. Two nodes $i$ and $j$ are called neighbors if $(i, j) \in \mathbf{E}$, and the set of neighbors of $i$ are denoted by $\mathcal{N}_{i}$.

In this paper we restrict ourselves to $D$-dimensional lattices as information graphs:

Definition 3 (D-dimensional lattice): A $D$-dimensional lattice, specifically a $n_{1} \times n_{2} \times \cdots \times n_{D}$ lattice, is a graph with $n_{1} n_{2} \ldots n_{D}$ nodes, in which the nodes are placed at the integer coordinate points of the $D$-dimensional Euclidean space and each real vehicle connects to vehicles which are exactly one unit away from it.

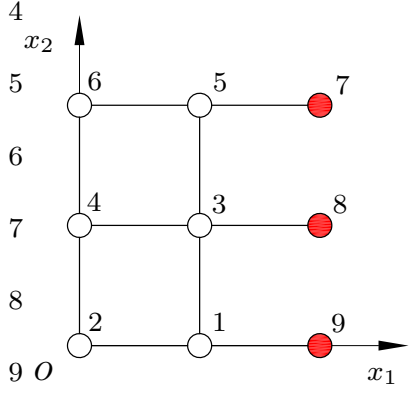

Fig. 4. A pictorial representation of a 2-D information graph. The filled node represent the reference vehicles and the solid lines represent edges in the information graph. 
Figure 4 depicts an example of 2-D lattice. A $D$-dimensional lattice is drawn in $\mathbb{R}^{D}$ with a Cartesian reference frame whose axes are denoted by $x_{1}, x_{2}, \ldots, x_{D}$. We also define $N_{d}(d=$ $1, \ldots, D)$ as the number of real vehicles in the $x_{d}$ direction. Then we have $N_{1} N_{2} \cdots N_{D}=N$ and $n_{1} n_{2} \ldots n_{D}=N+N_{r}$. An information graph is said to be square if $N_{1}=N_{2}=\cdots=N_{D}$. Note that the information graph for the vehicular platoon considered in the previous sections is a 1-D lattice with $N$ real vehicles (nodes) and $N_{r}(=1)$ reference vehicle.

For the ease of exposition, we only consider the case where the reference vehicles are arranged on one boundary of the lattice. Without loss of generality, let it be perpendicular to the $x_{1}$ axis, see Figure 4 for an example. This arrangement of reference vehicles simplifies the presentation of the results. Arrangements of reference vehicles on other boundaries of the lattice can also be considered, which does not significantly change the results; see [23], [24].

Due to its similarity with the 1-D case, we omit the details on desired separations etc, which are available in [2]. The control laws with RPAV and RPRV feedback, in terms of the errors $\tilde{p}_{i}$ are, respectively

$$
\begin{aligned}
u_{i}= & -\sum_{d=1}^{D} k_{\left(i, i^{d+}\right)}\left(\tilde{p}_{i}-\tilde{p}_{i^{d+}}\right)-\sum_{d=1}^{D} k_{\left(i, i^{d-}\right)}\left(\tilde{p}_{i}-\tilde{p}_{i^{d-}}\right)-b_{i} \dot{\tilde{p}}_{i} \\
u_{i}= & -\sum_{d=1}^{D} k_{\left(i, i^{d+}\right)}\left(\tilde{p}_{i}-\tilde{p}_{i^{d+}}\right)-\sum_{d=1}^{D} k_{\left(i, i^{d-}\right)}\left(\tilde{p}_{i}-\tilde{p}_{i^{d-}}\right) \\
& -\sum_{d=1}^{D} b_{\left(i, i^{d+}\right)}\left(\dot{\tilde{p}}_{i}-\dot{\tilde{p}}_{i^{d+}}\right)-\sum_{d=1}^{D} b_{\left(i, i^{d-}\right)}\left(\dot{\tilde{p}}_{i}-\dot{\tilde{p}}_{i^{d-}}\right)
\end{aligned}
$$

where $i^{d+}$ (respectively, $i^{d-}$ ) denotes the neighbor of $i$ on the positive (respectively, negative) $x_{d}$ axis. The closed loop dynamics are again represented as $\dot{x}=A^{(\mathrm{RPAV} \text { or RPR })} x$, where the state $x:=\left[\tilde{p}_{1}, \dot{\tilde{p}}_{1}, \cdots, \tilde{p}_{N}, \dot{\tilde{p}}_{N}\right] \in \mathbb{R}^{2 N}$ is a vector of the relative positions $\tilde{p}_{i}$ and relative velocities $\dot{\tilde{p}}_{i}$. The stability margin is defined as before.

It is shown in [2] that asymmetry in control gains can improve the stability margin with RPAV feedback, but the analysis is limited for $\epsilon \rightarrow 0$ and the case with RPRV feedback was not considered. In this paper, we consider the following homogeneous and asymmetric control gains that introduce asymmetry only in the $x_{1}$ axis:

RPAV:

$$
\begin{aligned}
& k_{\left(i, i^{1+}\right)}=(1+\epsilon) k_{0}, k_{\left(i, i^{1-}\right)}=(1-\epsilon) k_{0}, \\
& k_{\left(i, i^{d+}\right)}=k_{0},(d>1), b_{i}=b_{0} .
\end{aligned}
$$




$$
\begin{aligned}
k_{\left(i, i^{1+}\right)} & =(1+\epsilon) k_{0}, \quad k_{\left(i, i^{1-}\right)}=(1-\epsilon) k_{0}, \\
\text { RPRV: } \quad b_{\left(i, i^{1+}\right)}=(1+\epsilon) b_{0}, & b_{\left(i, i^{1-}\right)}=(1-\epsilon) b_{0}, \\
& k_{\left(i, i^{d+}\right)}=k_{0}, \quad b_{\left(i, i^{d+}\right)}=b_{0}, \quad(d>1) .
\end{aligned}
$$

Again, we comment that heterogeneity in control gains has little effect on the scaling trend of stability margin, not only for vehicular formation with lattice graphs but also for general graphs with bounded degree and weights, please refer to Lemma 2 given in the appendix.

We first summarize the results in [2], [23].

Proposition 2: Consider a vehicular formation whose information graph is a $D$-dimensional lattice. With the control gains given in (25) and (26) respectively.

1) [Theorem 1 of [2], Theorem 4 of [23]] With symmetric control $(\epsilon=0)$, both $S^{(\mathrm{RPAV})}$ and $S^{(\mathrm{RPRV})}$ are $O\left(\frac{1}{N_{1}^{2}}\right)$.

2) [Theorem 2 of [2]] With the control gains given by (25), the stability margin with RPAV feedback is $S^{(\mathrm{RPAv})}=O\left(\frac{\epsilon}{N_{1}}\right)$, which hold in the limit $\epsilon \rightarrow 0$ and $N_{1} \rightarrow \infty$.

We next state the main result of this section, which is a corollary of Theorem 1. It describes the stability margin for a vehicular formation with $D$-dimensional lattice information graph with asymmetric control.

Corollary 1: With the control gains given in (25) and (26) respectively, and $0<\epsilon<1$, the stability margin of the vehicular formation with RPAV or RPRV feedback is bounded away from 0 , uniformly in $N$. Specifically,

$$
\begin{aligned}
S^{(\mathrm{RPAV})} & \geq \frac{\Re\left(b_{0}-\sqrt{b_{0}^{2}-8 k_{0}\left(1-\sqrt{1-\epsilon^{2}}\right)}\right)}{2}, \\
S^{(\mathrm{RPRV})} & \geq \min \left\{b_{0}\left(1-\sqrt{1-\epsilon^{2}}\right), \frac{k_{0}}{b_{0}}\right\} .
\end{aligned}
$$

Remark 2: From Proposition 2, we see that with the particular arrangement of the reference vehicles as mentioned before, the stability margin of the vehicular formation with symmetric control only depend on $N_{1}$, the number of real vehicles along the $x_{1}$ axis of the information graph. For a square information graph, no matter how large its dimension $D$ is, the loss of stability margin with increasing number of vehicle $N$ is inevitable, since $N_{1}=N^{1 / D}$. To make the stability margin independent of $N$ with symmetric control, one needs to employ a non-square information graph, such that $N_{1}$ is a constant regardless of the increasing of $N$. The price one 
pays is either long range communication and/or increased number of reference vehicles; see [2], [23] for more details. In addition, for the RPAV feedback case, with vanishingly small amount of asymmetry, the stability margin is improved to $O\left(1 / N_{1}\right)$, compared to the $O\left(1 / N_{1}^{2}\right)$ trend in the symmetric case.

In contrast, Corollary 1 shows that with judicious asymmetric control, the stability margin can be made independent of the number of vehicles $N$ in the formation, without using the non-square information graph aforementioned. Note that the result we establish in this paper (Corollary 1) is stronger than that in [2], even though the control law is the same. The reason is that the analysis in [2] relied on a perturbation technique, which limited its applicability to vanishingly small $\epsilon$. In this paper we do not use perturbation techniques, and obtain result for any non-vanishing $\epsilon \in(0,1)$. In addition, we also consider the RPRV feedback case, while [2] analyzed only RPAV feedback.

Proof of Corollary 1$]$ With the control gains specified in (25) and (26) respectively, it is straightforward - through a bit tedious - to show that the state matrices $A^{(\mathrm{RPAV})}$ and $A^{(\mathrm{RPRV})}$ can be expressed in the following forms,

$$
\begin{aligned}
& A^{(\mathrm{RPAV})}=I_{N} \otimes A_{1}+L^{(D)} \otimes A_{2}, \\
& A^{(\mathrm{RPRV})}=I_{N} \otimes A_{3}+L^{(D)} \otimes A_{4},
\end{aligned}
$$

where $A_{1}, A_{2}, A_{3}, A_{4}$ are given in (10) and $L^{(D)}$ has the following form:

$$
L^{(d)}=I_{N_{d}} \otimes L^{(d-1)}+T^{(d)} \otimes I_{N_{1} N_{2} \cdots N_{d-1}}, \quad 2 \leq d \leq D,
$$

where $L^{(1)}$ is given in (11) and $T^{(d)}$ is a matrix of dimension $N_{d} \times N_{d}$, which is given by

$$
T^{(d)}=\left[\begin{array}{ccccc}
1 & -1 & & & \\
-1 & 2 & -1 & & \\
& \ddots & \ddots & \ddots & \\
& & -1 & 2 & -1 \\
& & & -1 & 1
\end{array}\right] .
$$

The eigenvalues of $T^{(d)}$ are given by (see [21]):

$$
\lambda_{\ell_{d}}=2-2 \cos \frac{\left(\ell_{d}-1\right) \pi}{N_{d}}, \quad \ell_{d}=1,2, \ldots, N_{d} .
$$


For example, for a 2-dimensional information graph shown in Figure 4.

$$
L^{(2)}=\left[\begin{array}{cccccc}
3 & -1+\epsilon & -1 & 0 & 0 & 0 \\
-1-\epsilon & 2+\epsilon & 0 & -1 & 0 & 0 \\
-1 & 0 & 4 & -1+\epsilon & -1 & 0 \\
0 & -1 & -1-\epsilon & 3+\epsilon & 0 & -1 \\
0 & 0 & -1 & 0 & 3 & -1+\epsilon \\
0 & 0 & 0 & -1 & -1-\epsilon & 2+\epsilon
\end{array}\right] .
$$

It's straightforward to show that $L^{(2)}=I_{3} \otimes L^{(1)}+T^{(2)} \otimes I_{2}$, where $T^{(2)}$ is a matrix with dimension $3 \times 3$.

From the proof of Lemma 1 , we see that the eigenvalues of $A^{(\mathrm{RPAV})}$ and $A^{(\mathrm{RPRV})}$ are given by the roots of the characteristic equations $s^{2}+b_{0} s+k_{0} \lambda_{\vec{\ell}}=0$ and $s^{2}+b_{0} \lambda_{\vec{\ell}} s+k_{0} \lambda_{\vec{\ell}}=0$ respectively, where $\lambda_{\vec{\ell}}$ is the eigenvalue of $L^{(D)}$, and $\vec{\ell}=\left(\ell_{1}, \cdots, \ell_{D}\right)$ in which $\ell_{d} \in\left\{1,2, \cdots, N_{d}\right\}$. We next claim that the eigenvalues of $L^{(D)}$ are given by

$$
\lambda_{\vec{\ell}}=\lambda_{\ell_{1}}\left(L^{(1)}\right)+\sum_{d=2}^{D} \lambda_{\ell_{d}}\left(T^{(d)}\right) .
$$

We prove by induction method. For the case $d=2, L^{(2)}=I_{N_{2}} \otimes L^{(1)}+T^{(2)} \otimes I_{N_{1}}$. Following (16) in the proof of Lemma 1, the eigenvalues of $L^{(2)}$ are given by

$$
\begin{aligned}
\lambda_{\ell_{1}, \ell_{2}} & =\bigcup_{\lambda_{\ell_{2}} \in \sigma\left(T^{(2)}\right)}\left\{\sigma\left(L^{(1)}+\lambda_{\ell_{2}} I_{N_{1}}\right)\right\} \\
& =\lambda_{\ell_{1}}\left(L^{(1)}\right)+\lambda_{\ell_{2}}\left(T^{(2)}\right),
\end{aligned}
$$

Now, we assume the general formula for the eigenvalues of $L^{(D-1)}$ is given by

$$
\lambda_{\ell_{1}, \ldots, \ell_{D-1}}=\lambda_{\ell_{1}}\left(L^{(1)}\right)+\sum_{d=2}^{D-1} \lambda_{\ell_{d}}\left(T^{(d)}\right) .
$$

For the case $d=D$, the matrix $L^{(D)}$ has the form given in (30), use (16) again, we have

$$
\begin{aligned}
\lambda_{\ell_{1}, \ldots, \ell_{D}} & =\bigcup_{\lambda_{\ell_{D}} \in \sigma\left(T^{(D)}\right)}\left\{\sigma\left(L^{(D-1)}+\lambda_{\ell_{D}} I_{N_{1} \cdots N_{D-1}}\right)\right\} \\
& =\lambda_{\ell_{1} \cdots \ell_{D-1}}\left(L^{(D-1)}\right)+\lambda_{\ell_{D}}\left(T^{(D)}\right),
\end{aligned}
$$

which proves the claim. Now, use (14) and (32), the smallest eigenvalue of $L^{(D)}$ is equal to $\lambda_{1}$, the smallest eigenvalue of $L^{(1)}$. The result now follows from Lemma 1 and Theorem 1 . 


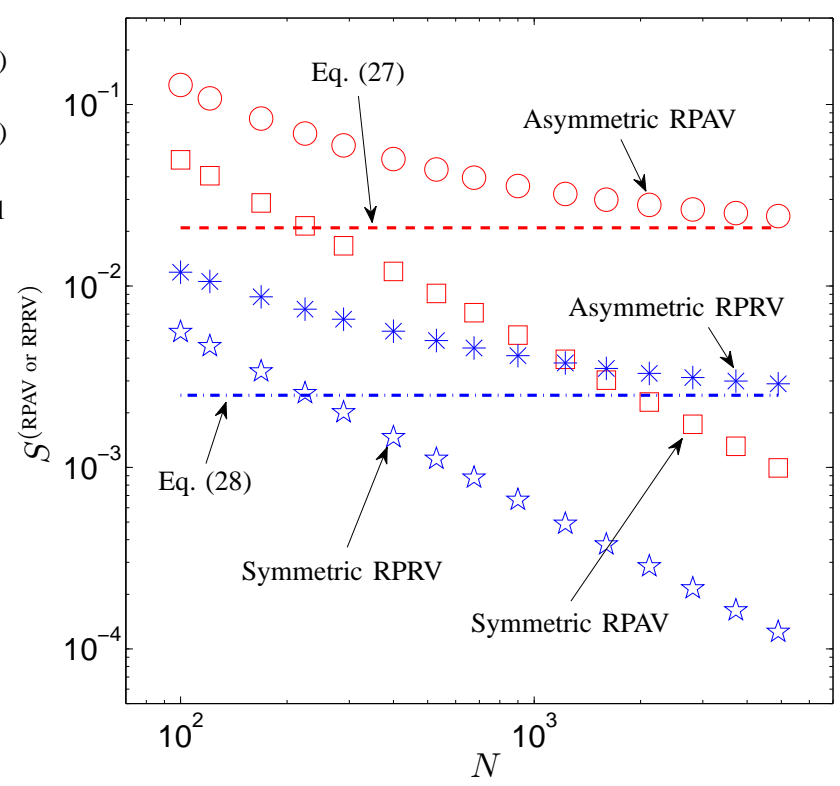

Fig. 5. Stability margin comparisons between symmetric control and asymmetric control for a vehicular formation with 2-D square information graph.

\section{A. Numerical verification for vehicular formation with D-dimensional information graph}

In this section, we present numerical verification of the theoretical predicted lower bounds of stability margin for vehicular formations with D-dimensional lattice information graphs. For simplicity, we take 2-D lattices as examples. We assume the information graph is square, i.e. $N_{1}=N_{2}=\sqrt{N}$. In addition, the stability margins with symmetric control are also computed to compare with the asymmetric case. For both symmetric and asymmetric controls, the nominal control gains used are $k_{0}=1, b_{0}=0.5$, and for asymmetric control, the amount of asymmetry used is $\epsilon=0.1$. We observe from Figure 5 that, with asymmetric control, the stability margin of the vehicular formation with RPAV or RPRV feedback is indeed uniformly bounded below by the prediction Eq. (27) and Eq. (28) respectively. Furthermore the stability margin with asymmetric control is much larger than that with symmetric control for the same $N$. 


\section{Summary}

We studied the stability margin of vehicular formations on lattice graphs with distributed control. The control signal at every vehicle depends on the measurements from its neighbors in the information graph, which is a $D$-dimensional lattice. Inspired by the previous works [1], [2], we examined the role of asymmetry in the control gains on the closed loop stability margin. We showed that with judicious asymmetry in the control gains, the stability margin of the vehicular formation can be bounded away from 0 uniformly in $N$. This eliminates the loss of stability margin with increasing $N$ that is seen with symmetric control. In this paper, the analysis of the stability margin avoids the PDE approximation and perturbation method used in [1], [2]. In particular, the latter limited the analyses in those papers to vanishingly small amount of asymmetry and resulted a $O(1 / N)$ scaling trend of stability margin. In addition, the control laws examined in [1], [2] required vehicles to have access to the desired velocity of the formation. We generalized the results to the case when only relative velocity and relative position measurements are available. We showed in this paper that in both cases (i.e., with or without absolute velocity feedback), stability margin can be made independent of the size of the formation with asymmetric control. The issue of sensitivity to external disturbances with asymmetric control is a topic of future research.

\section{REFERENCES}

[1] P. Barooah, P. G. Mehta, and J. P. Hespanha, "Mistuning-based decentralized control of vehicular platoons for improved closed loop stability," IEEE Transactions on Automatic Control, vol. 54, no. 9, pp. 2100-2113, September 2009.

[2] H. Hao, P. Barooah, and P. G. Mehta, "Stability margin scaling laws of distributed formation control as a function of network structure," IEEE Transactions on Automatic Control, vol. 56, pp. 923 - 929, April 2011.

[3] J. K. Hedrick, M. Tomizuka, and P. Varaiya, "Control issues in automated highway systems," IEEE Control Systems Magazine, vol. 14, pp. 21 - 32, December 1994.

[4] A. Okubo, "Dynamical aspects of animal grouping: swarms, schools, flocks, and herds," Advances in Biophysics, vol. 22, pp. 1-94, 1986.

[5] E. Wagner, D. Jacques, W. Blake, and M. Pachter, "Flight test results of close formation flight for fuel savings," in AIAA Atmospheric Flight Mechanics Conference and Exhibit, 2002, AIAA-2002-4490.

[6] S. Darbha and P. R. Pagilla, "Limitations of employing undirected information flow graphs for the maintenance of rigid formations for heterogeneous vehicles," International journal of engineering science, vol. 48, no. 11, pp. 1164-1178, 2010.

[7] A. Das, R. Fierro, V. Kumar, J. Ostrowski, J. Spletzer, and C. Taylor, "A framework for vision based formation control," IEEE Transactions on Robotics and Automation, vol. 18, no. 5, pp. 813-825, 2002.

[8] H. Tanner, G. Pappas, and V. Kumar, "Leader-to-formation stability," Robotics and Automation, IEEE Transactions on, vol. 20, no. 3, pp. 443-455, 2004. 
[9] S. Darbha and J. K. Hedrick, "String stability of interconnected systems," IEEE Transactions on Automatic Control, vol. 41, no. 3, pp. 349-356, March 1996.

[10] P. Seiler, A. Pant, and J. K. Hedrick, "Disturbance propagation in vehicle strings," IEEE Transactions on Automatic Control, vol. 49, pp. 1835-1841, October 2004.

[11] B. Bamieh, M. R. Jovanović, P. Mitra, and S. Patterson, "Effect of topological dimension on rigidity of vehicle formations: fundamental limitations of local feedback," in Proceedings of the 47th IEEE Conference on Decision and Control, Cancun, Mexico, 2008, pp. 369-374.

[12] M. R. Jovanović and B. Bamieh, "On the ill-posedness of certain vehicular platoon control problems," IEEE Trans. Automatic Control, vol. 50, no. 9, pp. 1307-1321, September 2005.

[13] F. Tangerman and J. Veerman, "Asymmetric Decentralized Flocks," accepted to IEEE Transactions on Automatic Control, 2011. [Online]. Available: http://www.mth.pdx.edu/ veerman/publ04.html

[14] H. Hao and P. Barooah, "Control of large 1D networks of double integrator agents: role of heterogeneity and asymmetry on stability margin," in IEEE Conference on Decision and Control, December 2010.

[15] J. Veerman, B. Stošić, and F. Tangerman, “Automated traffic and the finite size resonance," Journal of Statistical Physics, vol. 137, no. 1, pp. 189-203, October 2009.

[16] S. Darbha and J. K. Hedrick, "String stability of interconnected systems," IEEE Transactions on Automatic Control, vol. 41, no. 3, pp. 349-356, March 1996.

[17] Y. Zhang, B. Kosmatopoulos, P. Ioannou, and C. Chien, "Using front and back information for tight vehicle following maneuvers," IEEE Transactions on Vehicular Technology, vol. 48, no. 1, pp. 319-328, 1999.

[18] R. Middleton and J. Braslavsky, "String instability in classes of linear time invariant formation control with limited communication range," IEEE Transactions on Automatic Control, vol. 55, no. 7, pp. 1519-1530, 2010.

[19] S. Darbha, J. Hedrick, C. Chien, and P. Ioannou, "A comparison of spacing and headway control laws for automatically controlled vehicles," Vehicle System Dynamics, vol. 23, no. 8, pp. 597-625, 1994.

[20] S. Stankovic, M. Stanojevic, and D. Siljak, "Decentralized overlapping control of a platoon of vehicles," Control Systems Technology, IEEE Transactions on, vol. 8, no. 5, pp. 816-832, 2000.

[21] W. Yueh and S. Cheng, "Explicit eigenvalues and inverses of tridiagonal toeplitz matrices with four perturbed corners," The Australian \& New Zealand Industrial and Applied Mathematics (Anziam) Journal, vol. 49, no. 3, pp. 361-388, 2008.

[22] J. Veerman, G. Lafferriere, J. Caughman, and A. Williams, "Flocks and formations," Journal of Statistical Physics, vol. 121, no. 5, pp. 901-936, 2005.

[23] H. Hao, P. Barooah, and J. J. P. Veerman, "Effect of network structure on the stability margin of vehicle formation with distributed control," in IEEE Conference on Decision and Control, December 2010, pp. 4783 - 4788.

[24] H. Hao, P. Barooah, and P. G. Mehta, "Distributed control of two dimensional vehicular formations: stability margin improvement by mistuning," in ASME Dynamic Systems and Control Conference, October 2009, pp. 699-706.

[25] S. K. Yadlapalli, S. Darbha, and K. R. Rajagopal, "Information flow and its relation to stability of the motion of vehicles in a rigid formation," IEEE Transactions on Automatic Control, vol. 51, no. 8, August 2006.

[26] P. Barooah and J. Hespanha, "Graph effective resistance and distributed control: Spectral properties and applications," in Decision and Control, 2006 45th IEEE Conference on. IEEE, 2006, pp. 3479-3485.

[27] H. Khalil, Nonlinear Systems 3rd. Prentice hall Englewood Cliffs, NJ, 2002. 


\section{APPENDIX}

Lemma 2: Consider a vehicular formation whose information graph is an arbitrary connected graph (with 1 grounded node/leader) with bounded degree and weights. With the double integrator dynamics (1) and the following heterogeneous and symmetric control gains with RPAV and RPRV feedback respectively,

$$
\begin{aligned}
& u_{i}^{(\mathrm{RPAV})}=-\sum_{j \in \mathcal{N}_{i}} k_{0} w_{i, j}\left(\tilde{p}_{i}-\tilde{p}_{j}\right)-b_{0} \dot{\tilde{p}}_{i}, \\
& u_{i}^{(\mathrm{RPRV})}=-\sum_{j \in \mathcal{N}_{i}} k_{0} w_{i, j}\left(\tilde{p}_{i}-\tilde{p}_{j}\right)-\sum_{j \in \mathcal{N}_{i}} b_{0} w_{i, j}\left(\dot{\tilde{p}}_{i}-\dot{\tilde{p}}_{j}\right),
\end{aligned}
$$

where $k_{0}, b_{0}$ are positive constants and $w_{i, j}=w_{j, i}>0$ are the weight on the edge $(i, j)$ and $(j, i)$ respectively. The stability margin decays to zero as $N$ goes to infinity, i.e.

$$
S^{(\mathrm{RPAV} \text { or RPRV })} \rightarrow 0 \quad \text { as } \quad N \rightarrow \infty,
$$

where $N$ is the number of vehicles in the formation.

Proof of Lemma 2 The state matrices $A^{(\mathrm{RPAV})}$ and $A^{(\mathrm{RPRV})}$ can be expressed in the following forms,

$$
\begin{aligned}
& A^{(\mathrm{RPAV})}=I_{N} \otimes A_{1}+L \otimes A_{2}, \\
& A^{(\mathrm{RPRV})}=I_{N} \otimes A_{3}+L \otimes A_{4},
\end{aligned}
$$

where $A_{1}, A_{2}, A_{3}, A_{4}$ are given in (10) and $L$ is the grounded graph Laplacian of the vehicular formation. First, we recall that the Laplacian matrix $\mathcal{L}$ of an arbitrary graph $\mathrm{G}$ with edge weights $w_{i, j}$ is defined as

$$
\mathcal{L}_{i, j}= \begin{cases}-w_{i, j} & i \neq j,(i, j) \in \mathbf{E} \\ \sum_{k=1}^{N} w_{i, k} & i=j,(i, k) \in \mathbf{E} \\ 0 & \text { otherwise. }\end{cases}
$$

The grounded graph Laplacian $L$ is obtained by deleting the row and column of $\mathcal{L}$ corresponding to the leader (recall that we have a leader/reference vehicle). Without loss of generality, let it be indexed by 1 . The neighbors of the leader is denoted by $\mathcal{N}_{1}$.

From the proof of Lemma 1, we notice that the stability margin of the formation is determined by $\lambda_{1}$, the smallest eigenvalue of its grounded graph Laplacian $L$. 
We first claim that as long as its grounded graph Laplacian $L$ is symmetric, i.e. $w_{i, j}=w_{j, i}$, the smallest eigenvalue $\lambda_{1}$ of $L$ satisfies

$$
\lambda_{1} \rightarrow 0 \quad \text { as } \quad N \rightarrow \infty .
$$

This fact can be seen as a generalization of Lemma 3.2 of [25]. First of all, the grounded graph Laplacian $L$ is positive definite [26]. From the Rayleigh Ritz Theorem [27], for an arbitrary vector $x$, we have the following inequality

$$
x^{T} \lambda_{1}(L) x \leq x^{T} L x, \quad \Rightarrow \quad \lambda_{1} \leq \frac{x^{T} L x}{x^{T} x} .
$$

In particular, we pick the following vector with dimension $(N-1) \times 1, x=[1,1, \cdots, 1]^{T}$, then we have

$$
\lambda_{1} \leq \frac{x^{T} L x}{N-1} \leq \frac{\left|\mathcal{N}_{1}\right| \max _{j \in \mathcal{N}_{1}} w_{j, 1}}{N-1}
$$

where $\left|\mathcal{N}_{1}\right|$ denotes the number of neighbors of the leader. Since the weight $w_{j, 1}$ and $\left|\mathcal{N}_{1}\right|$ are bounded, when $N$ goes to infinity, the fact follows. With this, the rest of the proof follows from Lemma 1. 